\title{
CONSIDÉRATIONS SUR LES MODĖLES D'AVANT PROJET POUR CLASSES DE TARIF
}

\author{
ED. Franckx \\ Bruxelles
}

\section{CONSIDÉRATIONS PRÉLIMINAIRES}

a) L'assurance est un jeu. Le rôle principal de l'actuaire est d'aider l'assureur à ,trouver son adversaire". En assurance-vie, le risque est défini par la table de mortalité et l'assuré est trouvé quant il déclare son âge et son état de santé. En assurance non life, la question n'est pas aussi simple. Mais la pratique commerciale a introduit la notion de classe du tarif. Les risques qui appartiennent à une classe de tarif n'appartiennent pas à une classe homogène, mais les conséquences d'un sinistre sont comparables au sens du calcul des probalités. La valeur moyenne d'un sinistre résultant de la classe de tarif est statistiquement stable. Ce qui fondamentalement a garanti le fonctionnement de l'assurance non life est l'application de la loi des grands nombres. La régularité statistique sera toujours l'élement de classement et de décision. Nous considérons donc la classe de tarif comme étant l'élément primaire du riskbusiness, même si la valeur moyenne d'un sinistre varie dans le temps.

b) Etablir un modèle est toujours une approximation plus au moins bien réussie de la réalité. Le problème de la représentation d'un risque couvert par un contrat d'assurance ou un ensemble de contrats (réassurance) est un problème théorique. Autre chose est le problème de l'efficacité c'est-à-dire la possibilité d'effectuer des calculs pratiques. Le modèle gaussien de la loi des erreurs de mesure n'est pas theoriquement exact, mais il permet les calculs de compensation. Le modèle de Dodson en assurance-vie est une première approximation de la réalité mais il permet à l'actuaire d'effectuer ses tarifications.

Un souhait à formuler en non life, est de trouver des modèles non sophistiqués efficaces. 
c) Un modèle n'a pas nécessairement une expression mathématique. Il résulte d'un choix d'axiomes ou d'hypothèses, base à partir desquelles on déduit des règles de comportement, qui en découlent rationnellement. La pratique teste la valeur d'un modèle, elle se mesure par le fait qu'il permet de prévoir, en laissant des bénéfices à l'exploitant. Pour cela il doit nécessairement pouvoir résoudre numériquement un certain nombre de problèmes ,type”.

2. LE MODÈle MINIMAL OU LE MOdÈLE D'AVANT PROJET

a) Le modèle à priori

Quelque soit le modèle, il faut définir le processus de risque subi par contrat. On acceptera sous le modèle d'avant-projet les axiomes suivants:

A I la période de référence est constante - généralement l'exercice annuel

A2 l'arrivée d'un accident est défini par une loi de Poisson de paramêtre $\lambda_{t}$.

A3 chaque accident donne lieu à un paiement unitaire (on pratique la valeur moyenne).

Il en résulte que si la classe de tarif est constituée du $N_{t}$ risques identiques au cours de l'exercice et la fonction de répartition du total à payer $M$ est donné pour $m_{0}$ entier par

$$
\text { prob. }\left\{M \leq m_{0}\right\}=\sum_{1}^{m_{0}} \frac{\left(\lambda_{t} N_{t}\right)^{l}}{l !} \cdot e^{-\lambda_{t} N_{t}}
$$

et les tables de Poisson permettent de résoudre numériquement tous les problèmes pratiques y compris les problèmes de stop-loss.

b) Le problème statistique, la méthode du bilan et la procédure de prolongement

Tout revient à determiner le paramêtre de la loi de Poisson. On utilise la méthode du maximum de vraisemblance.

Si au cours du temps, on a constaté que la classe de tarif avait:

les populations:

$N_{0}, N_{1}, \ldots \ldots, N_{t-1}$

les sinistres:

$n_{0}, n_{1}, \ldots \ldots, n_{t-1}$ 
on trouvera que la meilleure estimation après $t$ exercices:

$$
\lambda^{*}=\frac{\sum_{0}^{t-1} n_{\mathrm{v}}}{\sum_{0}^{t-1} N_{\mathrm{v}}}
$$

et la méthode de decision revient à choisir pour le $i^{e}$ exercice:

$$
\lambda_{t}=\lambda^{*}
$$

La méthode conduit à déterminer le taux de prime suivant la „,toute vieille méthode du bilan".

En effet, le risque est représenté par un processus de Poisson de paramêtre $\lambda^{*}$ donc la prime à demander la valeur moyenne $\lambda^{*}$ est telle qu'elle équilibre sur la statistique globale du passé, dépenses et recettes constatées. En fait, on ne fait que ,prolonger ce taux de prime, par le processus de Poisson, qui a même valeur moyenne". C'est une méthode rudimentaire d'ou le nom du modèle minimal, ou modèle d'avant projet. Mais tout modèle de risque doit réaliser un processus du prolongement possédant une propriété isomorphe (propriété de comptabilité).

c) La formule de credibility

La méthode de comportement doit être permanente (principe de continuité) on aura donc:

$$
\left.\begin{array}{l}
\lambda_{t} \cdot \sum_{0}^{t-1} N_{v}=\sum_{0}^{t-1} n_{v} \\
\lambda_{t+1} \cdot \sum_{0}^{t} N_{v}=\sum_{0}^{1} n_{v}
\end{array}\right\} \rightarrow \lambda_{t+1}=\lambda_{t} \cdot \frac{\sum_{0}^{t-1} N_{v}}{\sum_{0}^{t} N_{v}}+\frac{n_{t}}{N_{t}} \cdot \frac{N_{t}}{\sum_{0}^{t} N_{v}}
$$

ce qui donne la formule de la credibility américaine

$$
\begin{aligned}
\lambda_{t+1} & =\frac{n_{t}}{N_{t}} \cdot \theta_{t}+\lambda_{t}\left(\mathrm{I}-\theta_{t}\right) \\
\theta_{t} & =\frac{N_{t}}{\sum_{v}^{t} N_{v}}
\end{aligned}
$$

$n_{t} / N_{t}$ est le taux de prime du dernier exercice. 


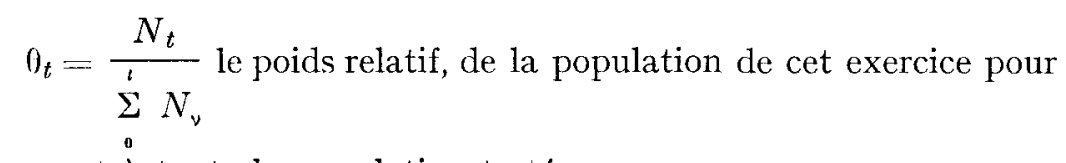
rapport à toute la population testée.

La formule $(4, a)$ est avant tout une règle d'adaptation, donc un règle dynamique. On peut la justifier de bien d'autres manières en général, bien plus compliquées.

\section{d) Problème type}

A partir d'un modèle désigné on doit pouvoir résoudre numériquement un nombre de problèmes type qui sont toujours les mêmes car les problèmes que la pratique soulève sont indépendants de la structure du modèle désigné. Citons: problèmes de bonus, problèmes d'excess of' loss et stop loss, problèmes de décisions et de comportement, problèmes d'évolution des fonds de garantie et problème de ruine, problèmes de solvabilité. Avec comme référence le modèle d'avantprojet, certaines de ces questions ont été évoquées dans le Bulletin de l'Association Belge des Actuaires et dans les bulletins d'ASTIN.

Mais il nous semble plus important sur le plan théorique, d'évoquer à Colchester une large extension du modèle d'avant-projet en introduisant la notion d'information dans le modèle et surtout la souplesse qui s'en suit.

\section{De la valeur de l'information}

a) Dans le modèle minimal on fait une hypothèse implicite: l'information du passé possède une valeur invariante; une observation s'il y a cinq ans a la même valeur que celle de la dernière année. Il n'en est rien en pratique et les brokers utilisent des échelles qui réduisent la valeur de l'information dans le temps, surtout en cas d'irregularité du marché de Londres. Cela revient à dire que l'information ne survit pas intégralement dans le temps. On va lui faire subir un processus d'usure.

On peut le définir en prenant une suite non croissante.

$$
v_{0}=\mathrm{I} \geq v_{-1} \geq v_{-2} \ldots \ldots \geq v_{-k} \ldots \geq 0
$$


ou $v_{0}$ est la valeur d'une observation au temps zéro, du dernier exercice ou $v_{-1}$ est la valeur d'une observation au temps zéro, de l'avantdernier exercice etc. .....

Par exemple, la méthode d'avant projet suppose: $\mathrm{I}=v_{0}=v_{-1}=$ $\ldots=v_{-k}=\ldots$ une méthode de ,non-confiance" serait donné par $v_{0}=\mathrm{I} v_{-1}=v_{-1}=\ldots=v_{-k}=0$ elle impliquerait que seuls sont pris en consideration les résultats du dernière exercice.

Le choix (5) est donc assez subjectif, il caractérise toutes les manières de comportement de l'assureur ou du marché.

b) Le problème statistique et variation sur la méthode du bilan

On peut toujours utiliser la méthode du maximum de vraisemblance mais à la fin du $t$ exercice il convient d'introduire ce qui survit des populations fin d'exercice,

aux populations

$N_{t-1}^{t-1}=v_{0} \cdot N_{t-1} \quad N_{t-2}^{t-1}=v_{-1} \cdot N_{t-2} \ldots \ldots N_{0}^{t-1}=v_{-t+1} \cdot N_{0}$ aux sinistres $n_{t-1}^{t-1}=v_{0} \cdot n_{t-1} \quad n_{t-2}^{t-1}=v_{-1} \cdot n_{t-1} \ldots \ldots n_{0}^{t-1}=v_{-t+1} \cdot n_{0}$.

La meilleure estimation après $t$ exercice sera:

$$
\lambda^{*}=\frac{\sum_{0}^{t-1} n_{v}^{t-1}}{\sum_{0}^{t-1} N_{v}^{t-1}}
$$

et la méthode de décision revient dans ce cas à choisir $\lambda_{t}=\lambda^{*}(3)$.

$\mathrm{La}$ formule (6) est une valeur moyenne pondérée. Gauss a introduit une formule analogue en Géodesie. Les Lloyds en font un large emploi. On utilise toujours la méthode du bilan, mais sur des chiffres ,conventionnellement aménagés par la loi (5)". C'est une méthode de „clefs" que les dirigeants utilisent aisément. Ici au point de vue risque, c'est le taux de prime ,pondéré" qu'on prolonge par un processus compatible de Poisson.

A partir de ce dernier on résout par la même technologie les problèmes ,type".

c) La formule généralisée de credibility

On peut se poser la question de savoir sous quelle forme se maintiennent les formules $(4 \mathrm{a}, 4 \mathrm{~b})$. 
En fait, on a, à tout moment, deux populations statistiques conventionnelles qui évoluent de manière similaire: celle des sinistres d'une part, celle des contrats d'autrepart. Théoriquement l'une et l'autre varient au cours de l'exercice à cause de la mortalité de l'information. C'est ce qui a été constaté par l'introduction des $N_{v}^{t-1}$ et $n_{v}^{t-1}$.

Dès lors on a, par la méthode du paragraphe précédent:

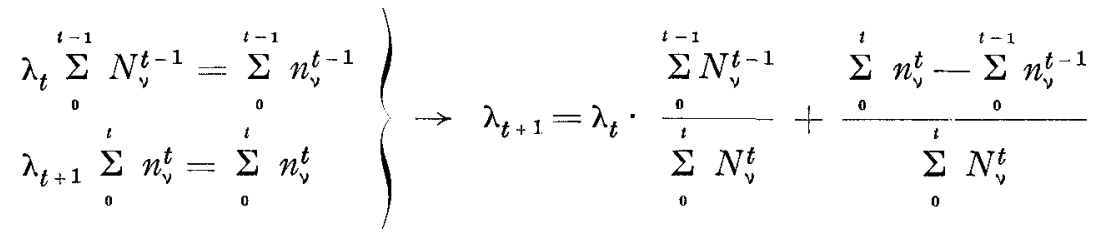

posons

$$
\begin{gathered}
\tilde{\lambda}_{t} \cdot \sum_{0}^{t-1} N_{v}^{t}=\lambda_{t} \cdot \sum_{0}^{t-1} N_{v}^{t-1} \\
\tilde{n}_{t}=n_{t}-\left(\sum_{0}^{t-1} n_{v}^{t}-\sum_{0}^{t-1} n_{v}^{t-1}\right)
\end{gathered}
$$

alors on garde toujours la même structure de credibility

$$
\left\{\begin{aligned}
\lambda_{t+1} & =\frac{\tilde{n}_{t}}{N_{t}^{t}} \theta_{t}+\tilde{\lambda}_{t}\left(\mathbf{I}-\theta_{t}\right) \\
\theta_{t} & =\frac{N_{t}^{t}}{\sum_{v}^{t} N_{v}^{t}}
\end{aligned}\right.
$$

$\tilde{\lambda}_{t}$ est la prime compensée, c'est celle que l'on aurait dû exiger de la population ,restante" pour maintenir la même rentrée de fonds.

$\tilde{n}_{t}$ est la valeur compensée des dépenses, si on admet que pour chaque sinistre ,éffacé" on rembourse la somme unitaire payée.

$\tilde{n}_{t} / N_{t}$ est par suite la valeur du taux de prime compensée au cours du dernier exercice. Ces questions ont été l'objet d'exposés à Amsterdam et à Trieste.

\section{Conclusions ou Considérations finales}

Notre but a été de mettre en lumière des méthodes simples, d'adaption qui permettent simultanément d'introduire un modéle 
simplifié de risque et sa modification en fonction de la valeur de l'information. La nécessité d'introduire des procédures à caractère dynamique nous semble essentielle en pratique. La recherche opérationnelle le prouve. Les procédés des actuaires de la Casualty Society le confirment. On doit se plier à la réalité des faits.

Mais il y a conceptuellement deux problèmes actuariels différents:

- D'une part l'établissement d'un modèle non statique du risque couvert.

- D'autre part la technologie uniforme, à élaboration plus au moins compliquée, qui permet de résoudre numériquement les problèmes ,"type”.

Même en assurance-vie la méthologie a admis cette séparation. Le premier problème est celui du choix d'une table de mortalité, le second comprend en particulier toute la technologie du calcul des primes et des réserves.

Mais comme en vie c'est l'efficacité du modèle de risque qui garantira sa survie. Donc une théorie non life réellement appicable demeure „per se" un objectif actuariel estimable. 